\title{
Effects of electrostimulation therapy in facial nerve palsy
}

\author{
Laura Sommerauer, Simon Engelmann, Marc Ruewe, Alexandra Anker, Lukas Prantl, \\ Andreas Kehrer \\ Department of Plastic, Hand and Reconstructive Surgery, University Medical Centre Regensburg, Regensburg, Germany
}

Facial palsy (FP) is a functional disorder of the facial nerve involving paralysis of the mimic muscles. According to the principle "time is muscle," early surgical treatment is tremendously important for preserving the mimic musculature if there are no signs of nerve function recovery. In a 49-year-old female patient, even 19 months after onset of FP, successful neurotization was still possible by a V-to-VII nerve transfer and cross-face nerve grafting. Our patient suffered from complete FP after vestibular schwannoma surgery. With continuous application of electrostimulation (ES) therapy, the patient was able to bridge the period between the first onset of FP and neurotization surgery. The significance of ES for mimic musculature preservation in FP patients has not yet been fully clarified. More attention should be paid to this form of therapy in order to preserve the facial musculature, and its benefits should be evaluated in further prospective clinical studies.

Keywords Facial paralysis / Electrical stimulation / Neurotization / Cross face nerve graft / Craniofacial
Correspondence: Laura Sommerauer Department of Plastic, Hand and Reconstructive Surgery, University Medical Centre Regensburg, FranzJosef-Strauss-Allee 11, 93053 Regensburg, Germany Tel: +49-941-944-14857 Fax: +49-941-944-6948 E-mail: laura.sommerauer@ukr.de

\section{Andreas Kehrer}

Department of Plastic, Hand and Reconstructive Surgery, University Medical Centre Regensburg, FranzJosef-Strauss-Allee 11, 93053 Regensburg, Germany Tel: +49-941-944-6763 Fax: +49-941-944-6948 E-mail: AndreasKehrer@gmx.de

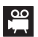

Received: May 31, 2020 • Revised: July 4, 2020 • Accepted: July 10, 2020

pISSN: 2234-6163 • elSSN: 2234-6171 • https://doi.org/10.5999/aps.2020.01025• Arch Plast Surg 2021;48:278-281

\section{INTRODUCTION}

Facial palsy (FP) is a functional disorder of the facial nerve involving paralysis of the mimic musculature. Irreversibility may be assumed if no signs of improvement occur within 18 months after the primary manifestation of FP. The symptoms include excruciating impairments, such as facial asymmetry, limited eye closure, oral incontinence, social dysfunction, and depression [13]. The prevalence ranges between 23 and 35 cases per 100,000, with Bell's palsy being the most common etiology in 50\%-75\% of cases [4]. Surgical therapy includes dynamic or static procedures, such as nerve transfer/transplantation, free muscle trans- plantation, transfer of regional motor units, eyelid implants, lower eyelid suspension, and static suspension with autologous tissue [5]. In this case report, we describe a surprising effect of electrostimulation (ES) applied in a case of complete FP.

\section{CASE}

A 49-year-old female physiotherapist suffered from complete left peripheral FP after resection of a vestibular schwannoma (World Health Organization grade I) in August 2015. Her House-Brackmann (HB) scale was grade VI. A 1.2 g platinum weight had been implanted in the left upper lid 8 days postoper- 
atively. The patient was offered a neurotization procedure, which she initially refused. Instead, the patient performed selftherapy using ES (Paresestim device; Krauth+Timmermann $\mathrm{GmbH}$, Hamburg, Germany). It included an electrical current with a pulse width not exceeding 100 milliseconds and a current strength of $10 \mathrm{~mA}$. The device guidelines were followed, with the goal of achieving maximum muscle contraction using the least current possible. A reasonable impulse is considered to range from 1 to 4 seconds, with 4 seconds being recommended if the patient can actively support the muscle movement. ES is suggested to be performed twice daily for 10 minutes. For 19 months, the patient stimulated her facial muscles one or two times daily for 10-30 minutes.

In March 2017, the patient presented at the department of plastic and reconstructive surgery at our hospital with longstanding FP. Lagophthalmos measured $6 \mathrm{~mm}$ after the previous upper eyelid implant. The patient complained of facial asymmetry, lack of oral competence, and facial expression. Nonetheless, remarkable facial tone was noted on the paralyzed side. A free muscle graft was recommended. However, after the patient reported being able to induce facial motion using the Paresestim device and demonstrated this ability, the operative plan was changed. Consequently, a neurotization procedure, including a V-to-VII nerve transfer (to the facial nerve trunk) and two crossface nerve grafts (CFNGs; eye branch and smile branch) with end-to-end coaptation, was planned 19 months after the primary manifestation of FP and performed by the last author in April 2017. During the operation, a certain degree of fatty degeneration of the zygomaticus major muscle was seen. ES was again conducted by the patient every other day for 10 minutes for 2-3 months postoperatively. After 4-5 months, the first improvements of symptoms and facial tone in repose were noticed (Fig. 1). With regard to synkinesis, slight contractions of the orbicu- laris oculi muscle while chewing were noticed by the patient. Lower lid reconstruction using a tarsal-strip procedure was performed in July 2017. Due to size progression of a residual tumor after her first surgery, re-craniotomy and complete tumor resection were deemed necessary in October 2017. Facial expression pre- and post-neurotization is demonstrated in Supplemental Video 1 . The patient provided informed consent for the use of all pictures or videos taken. The study was approved by the Institutional Review Board Committee of the University of Regensburg (reference number: 14-101-0251) and was designed in accordance with the Declaration of Helsinki.

\section{DISCUSSION}

The remarkable aspect of this case is the fact that the patient managed to maintain facial muscle function through ES for 19 months. Thus, even after this exceptionally long time, musclepreserving surgery with nerve transfer and CFNG could still be performed successfully.

Nevertheless, ES is one of the most controversial and non-evidence-based adjunctive therapies for FP [4]. The indications for ES have been described as incomplete posttraumatic or postoperative peripheral nerve lesions, muscle atrophy, hyperkinesis, or synkinesis $[2,4,6]$. A thorough review of the literature by our group demonstrated non-homogeneous recommendations for ES [7]. Another literature review by Teixeira et al. [8] to evaluate physical therapy for Bell's palsy was done in 2011. Three trials (86, 149, and 59 patients) studied the efficacy of ES, and could not prove benefits after 6 months. Yet, no statistically significant difference in synkinesis between patients with ES and a control group was found [9]. One study reported worse functional recovery with ES [9]. Faster recovery with ES was found by Flores, whereas Manikandan [9] described oppo-
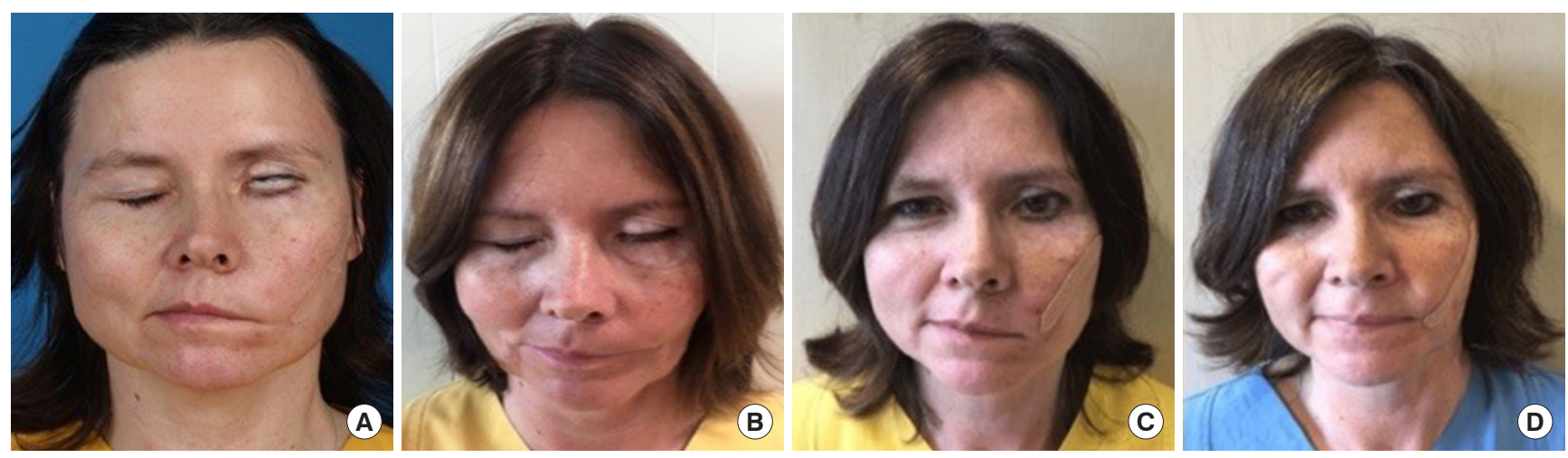

Fig. 1. Preoperative (A) and postoperative (B) photographs of eye closure: despite the eyelid weight, no eye closure was possible due to the paralyzed orbicularis oculi muscle preoperatively. Preoperative (C) and postoperative (D) photographs of smile: the patient's left face showed a clear improvement of muscle tension with a recovered nasolabial fold, a more centrally positioned philtrum, and an almost symmetrical smile with improvement of excursion of approximately $5 \mathrm{~mm}$ at 5 months after neurotization. 
site results. The authors of this review concluded that there is no high-quality evidence for the efficacy of any physical therapy in FP [8]. However, Tuncay et al. [10] analyzed the role of ES added to conventional therapy in $\mathrm{FP}$ in a randomized controlled trial (60 patients), which showed that patients receiving ES presented with improved functional facial movements compared to patients only treated with conventional therapy. Furthermore, ES is beneficial in chronic FP with delayed clinical improvement [7]. After muscle-replacement surgery in long-standing FP, ES of the muscle transplant is started after 6 weeks. As soon as an arbitrary contraction of the muscle transplant is possible, ES is stopped to prevent synkinesis [4]. In our case, the patient was able to partially preserve her original facial musculature through continuous application of ES. Consequently, neurotization of the remaining facial muscles was still successful after 19 months. Most likely due to the elapsed time between the onset of FP and the neurotization procedure, complete restoration of muscle function and symmetry was not possible.

Early treatment of irreversible FP, following the "time is muscle" principle, is of tremendous importance: after onset of FP without signs of adequate functional progression, neurotization should be performed as early as 6 months to preserve the mimic musculature [11-13]. Facial muscles may be preserved by nerve transfer and CFNG if performed in a timely manner. When bridging the period between the first onset of FP and musclepreserving surgery, atrophy of the mimic muscles may be attenuated by ES [4].

In contrast, after a period of 15-18 months without neural input, the muscle is irrevocably weakened and subject to fatty degeneration with atrophy. A multitude of studies have evaluated the best time for intervention for surgical reconstruction [1114].

Rivas et al. [13] developed a model for early prediction of facial nerve recovery after vestibular schwannoma surgery to select patients for facial reanimation surgery. They recommend surgery sooner than a waiting period of 1 year to improve outcomes. Their recovery model was tested by Albathi et al. [11], who selected patients after cerebellopontine angle tumor resection with an uninterrupted facial nerve for reanimation surgery after a postoperative observation period shorter than 12 months. Early surgical intervention shortened the duration of paralysis. Patients who refused facial reanimation surgery presented at best with $\mathrm{HB}$ grade $\mathrm{V}$ recovery after 18 months of follow-up, whereas patients with neurotization improved from $\mathrm{HB}$ grade VI to grade II or III in most cases. Therefore, the authors recommended facial reanimation as early as 6 months after FP. Successful rehabilitation is unlikely if there are no clinical signs of spontaneous nerve recovery in the first 6 months.
Further studies considering the best timing for facial reanimation and factors affecting the recovery of facial nerve function concluded that the only significant factor influencing the prognosis was the duration of the preoperative deficit. The most critical time for restoring function of $\mathrm{HB}$ grades III and IV is 6 months [14]. Patients who show no signs of recovery in the first 6 months, even if their facial nerves are intact, recover poorly and are candidates for early facial reanimation surgery. A strict indication for surgery according to the "time is muscle" principle is no improvement of at least 1 point on the HB scale by 6 months after the onset of FP following tumor resection [12].

The optimal time frame for facial reanimation surgery was long exceeded in our case. Therefore, complete functional restoration could not be achieved after the neurotization procedure. Nevertheless, the postoperative result was judged to be favorable with regard to the 19-month denervation period, which seemed attributable to continuous ES. According to our clinical experience, facial movement could not otherwise have been reestablished after 19 months of denervated facial musculature.

\section{NOTES}

\section{Conflict of interest}

No potential conflict of interest relevant to this article was reported.

\section{Ethical approval}

The study was approved by the Institutional Review Board of the University of Regensburg (IRB No. 14-101-0251) and performed in accordance with the principles of the Declaration of Helsinki. Written informed consent was obtained.

\section{Patient consent}

The patient provided written informed consent for the publication and the use of her images.

\section{Author contribution}

Conceptualization: all authors. Data curation: L Sommerauer. Formal analysis: L Sommerauer. Methodology: S Engelmann, M Ruewe, A Anker, L Prantl, A Kehrer. Project administration: L Sommerauer, M Ruewe, A Kehrer. Visualization: L Sommerauer, A Kehrer. Writing - original draft: L Sommerauer. Writing - review \& editing: A Kehrer.

\section{ORCID}

Laura Sommerauer https://orcid.org/0000-0002-3642-1451 Simon Engelmann https://orcid.org/0000-0003-3810-3307 Marc Ruewe https://orcid.org/0000-0002-6788-0853 
Alexandra Anker https://orcid.org/0000-0002-9004-073X

Lukas Prantl https://orcid.org/0000-0003-2454-2499

Andreas Kehrer https://orcid.org/0000-0001-9472-7662

\section{Supplementary material}

Supplemental Video 1. Video case report facial palsy. Supplemental data can be found at: https://doi.org/10.5999/aps.2020.01025. v001.

\section{REFERENCES}

1. Kehrer A, Engelmann S, Ruewe M, et al. Anatomical study of the zygomatic and buccal branches of the facial nerve: application to facial reanimation procedures. Clin Anat 2019; 32:480-8.

2. Eviston TJ, Croxson GR, Kennedy PG, et al. Bell's palsy: aetiology, clinical features and multidisciplinary care. J Neurol Neurosurg Psychiatry 2015;86:1356-61.

3. Kehrer A, Mandlik V, Taeger C, et al. Postoperative control of functional muscle flaps for facial palsy reconstruction: ultrasound guided tissue monitoring using contrast enhanced ultrasound (CEUS) and ultrasound elastography. Clin Hemorheol Microcirc 2017;67:435-44.

4. Paternostro-Sluga T, Herceg M, Frey M. Conservative treatment and rehabilitation in peripheral facial palsy. Handchir Mikrochir Plast Chir 2010;42:109-14.

5. Kehrer A, Engelmann S, Ruewe M, et al. Perfusion maintains functional potential in denervated mimic muscles in early persistent facial paralysis which requires early microsurgical treatment: the histoanatomic basis of the extratemporal facial nerve trunk assessing axonal load in the context of possible nerve transfers. Clin Hemorheol Microcirc 2018; 70:1-13.

6. Mosforth J, Taverner D. Physiotherapy for Bell's palsy. Br Med J 1958;2:675-7.

7. Buttress S, Herren K. Towards evidence based emergency medicine: best BETs from the Manchester Royal Infirmary. Electrical stimulation and Bell's palsy. Emerg Med J 2002; 19:428.

8. Teixeira LJ, Valbuza JS, Prado GF. Physical therapy for Bell's palsy (idiopathic facial paralysis). Cochrane Database Syst Rev 2011;(12):CD006283.

9. Manikandan N. Effect of facial neuromuscular re-education on facial symmetry in patients with Bell's palsy: a randomized controlled trial. Clin Rehabil 2007;21:338-43.

10. Tuncay F, Borman P, Taser B, et al. Role of electrical stimulation added to conventional therapy in patients with idiopathic facial (Bell) palsy. Am J Phys Med Rehabil 2015;94: 222-8.

11. Albathi M, Oyer S, Ishii LE, et al. Early nerve grafting for facial paralysis after cerebellopontine angle tumor resection with preserved facial nerve continuity. JAMA Facial Plast Surg 2016;18:54-60.

12. Boahene K. Facial reanimation after acoustic neuroma resection: options and timing of intervention. Facial Plast Surg 2015;31:103-9.

13. Rivas A, Boahene KD, Bravo HC, et al. A model for early prediction of facial nerve recovery after vestibular schwannoma surgery. Otol Neurotol 2011;32:826-33.

14. Ozmen OA, Falcioni M, Lauda L, et al. Outcomes of facial nerve grafting in 155 cases: predictive value of history and preoperative function. Otol Neurotol 2011;32:1341-6. 